This article may be downloaded for personal use only. Any other use requires prior permission of the author and AIP Publishing. The following article appeared in Halverson, T., louchtchenko, D., \& Roy, P.-N. (2018). Quantifying entanglement of rotor chains using basis truncation: Application to dipolar endofullerene peapods. Journal of Chemical Physics, 148(7), 074112 and may be found at https://doi.org/10.1063/1.5011769 


\title{
Quantifying entanglement of rotor chains using basis truncation: Application to dipolar endofullerene peapods
}

\author{
Tom Halverson, Dmitri louchtchenko, and Pierre-Nicholas Roy \\ Department of Chemistry, University of Waterloo, Waterloo, Ontario N2L 3G1, Canada
}

(Received 2 November 2017; accepted 5 February 2018; published online 21 February 2018)

\begin{abstract}
We propose a variational approach for the calculation of the quantum entanglement entropy of assemblies of rotating dipolar molecules. A basis truncation scheme based on the total angular momentum quantum number is proposed. The method is tested on hydrogen fluoride (HF) molecules confined in $\mathrm{C}_{60}$ fullerene cages themselves trapped in a nanotube to form a carbon peapod. The rotational degrees of freedom of the HF molecules and dipolar interactions between neighboring molecules are considered in our model Hamiltonian. Both screened and unscreened dipoles are simulated and results are obtained for the ground state and one excited state that is expected to be accessible via a far-infrared collective excitation. The effect of basis truncation on energetic and entanglement properties is examined and discussed in terms of size extensivity. It is empirically found that for unscreened dipoles, a total angular momentum cutoff that increases linearly with the number of rotors is required in order to obtain proper system size scaling of the chemical potential and entanglement entropy. Recent experiments [A. Krachmalnicoff et al., Nat. Chem. 8, 953 (2016)] suggest substantial screening of the HF dipole moment, so much smaller basis sets are required to obtain converged results in this realistic case. Static correlation functions are also computed and are shown to decay much quicker in the case of screened dipoles. Our variational results are also used to test the accuracy of perturbative and pairwise ansatz treatments. Published by AIP Publishing. https://doi.org/10.1063/1.5011769
\end{abstract}

\section{INTRODUCTION}

It has become self-evident that for computational technology to push past the inevitable breakdown of Moore's law, ${ }^{1}$ the use of quantum information devices (QIDs) will be necessary. ${ }^{2-4}$ The current model for the achievement of true QIDs is the utilization of entangled quantum states-i.e., a quantum system whose states cannot be expressed as a simple product. Most often, these candidate quantum systems are the spin states of single particles, e.g., bosons or fermions. These types of systems have proven to be quite robust but have two major drawbacks: (1) in most cases, they are limited to two levels and (2) they are difficult to construct outside of highly controlled laboratory settings. Most likely, (1) is a nonstarter since to date, binary switches have provided enough complexity to bring computer science to the point it is at today. On the other hand, it is unclear whether or not the exotic methods and cost prohibitive materials currently employed to study quantum entanglement will ever be viable for mass production, meaning (2) is the current source of immediate limitation. It is entirely possible that these issues will be too difficult to overcome, and it is, therefore, paramount that other candidates be explored. In particular, carbon fullerene-based nano-molecular assemblies (NMAs), which can be produced using available methodologies, could prove to be a viable alternative. ${ }^{5-13}$ One drawback of directly using NMAs is that one must then rely on the electronic states of the carbon structure as the medium for the desired process. However, the advent of endofullerenes-i.e., the confinement of an atom or molecule inside a closed fullerene structure — has added yet another level of tunable complexity to this pursuit. Specifically, "rotor-doped" NMAs, which are systems of endofullerenes, have become a subject of great interest. ${ }^{14-17}$ However, the study of confined molecules is relatively new, and only modest characterization of these systems has been done.

To underpin this pursuit, we present here a model Hamiltonian that is a first approximation to the type of confined molecule NMAs alluded to above, in a similar manner to that of the Bose-Hubbard model. ${ }^{18}$ In particular, the Hamiltonian described herein approximates confined molecules as a linear chain of pinned, rigid rotors with dipole-dipole interactions. A schematic representation of the model is shown in Fig. 1. The model contains parameters that can be tuned to simulate realistic molecular assemblies of many different types. To showcase its depth and breadth, we present results in two parametric regimes-high and low dipole coupling. We explore these two limits in the context of two physically realistic systems - the bare and screened dipole interactions of hydrogen fluoride (HF). Although similar assemblies of polar rotors have recently been examined for use as the basis of a quantum computer, ${ }^{19-22}$ in the present work, we include all the rotational degrees of freedom and do not truncate the system to a qubit representation.

Since our goal is the characterization of quantum information properties of these systems, the quantity in which we are interested is the entanglement entropy or, more formally, the von Neumann (vN) entropy $S_{A}$ of a given subsystem of the total NMA. Pragmatically, we subdivide the NMA into two parts, $A$ and $B$, and compute the vN entropy of $A$ 


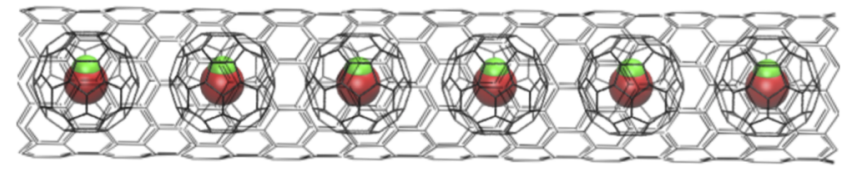

FIG. 1. Schematic representation of the peapod confined rotor model. The hydrogen and fluorine atoms composing the HF molecule are, respectively, represented as green and red van der Waals spheres.

relative to $B$. This yields a measurement of the degree to which $A$ and $B$ are entangled-i.e., how much information can be gleaned about $B$ from a measurement of $A$. In the quantum information world, this entanglement can then be used as a resource. ${ }^{23}$ Unfortunately, the journey from theoretical understanding of $S_{A}$ to a working QID is long, and, most likely, realistic QIDs will be comprised of much more complicated structures, but the information presented herein should provide a good starting point from which a robust framework can be built.

The remainder of this paper is organized as follows: Theoretical concepts are presented in Sec. II; results for energetic, entanglement, and correlation properties are given in Sec. III; we provide concluding remarks in Sec. IV.

\section{THEORY}

In the case of confined diatomic molecules inside a fullerene cage, it is not easy to say how much environmental interactions, e.g., cage deformations, will affect the low lying rovibrational spectra of the molecules contained therein. Although, it can be concluded from recent developments ${ }^{17}$ that, as a good first approximation, a linear NMA of this type can be treated as a chain of pinned rotors under the effect of screened dipole-dipole interactions. Due to this, we propose the following model Hamiltonian as a starting point for the characterization of angular momentum-based QIDs,

$$
\hat{H}=\alpha \sum_{i=1}^{n} \hat{l}_{i}^{2}+\beta \sum_{i<j} \hat{V}_{i j},
$$

where $n$ is the number of rotors in the chain, $\alpha$ and $\beta$ are the adjustable physical parameters, and

$$
\hat{V}_{i j}=\frac{\hat{x}_{i} \hat{x}_{j}+\hat{y}_{i} \hat{y}_{j}-2 \hat{z}_{i} \hat{z}_{j}}{(j-i)^{3}}
$$

is the dipole potential, with the chain axis aligned along the space-fixed $z$-axis. In the present case, the parameter $\alpha$ equals the rotational constant $B=\hbar^{2} /(2 I)$ where $I$ is the moment of inertia of the rotor, and the coupling $\beta=\mu^{2} /\left(4 \pi \epsilon_{0} R^{3}\right)$ depends on the dipole moment $\mu$ of the molecule and the distance $R$ between adjacent rotors.

We seek to compute the ground state $|0\rangle$ (with energy $E_{0}$ ) and the first non-degenerate excited state $|1\rangle$ (with energy $\left.E_{1}\right)$ via a variational basis set calculation. That is, we expand the Hamiltonian in a finite numerical basis and compute the eigenvalues and eigenvectors via numerical methods,

$$
\tilde{H}_{I J}=\langle I|\hat{H}| J\rangle .
$$

The basis set utilized is a direct product of free rotor eigenstates (spherical harmonics),

$$
|I\rangle=\bigotimes_{i=1}^{n}\left|l_{i} m_{i}\right\rangle=\left|l_{1} m_{1} l_{2} m_{2}, \ldots, l_{n} m_{n}\right\rangle,
$$

with

$$
\hat{l}_{k}^{2}\left|l_{k} m_{k}\right\rangle=l_{k}\left(l_{k}+1\right)\left|l_{k} m_{k}\right\rangle \text {. }
$$

Along with this, we also impose a specific basis truncation scheme, as to ease the exponential scaling of the direct product basis,

$$
\sum_{i=1}^{n} l_{i} \leq L
$$

The drastic effect of this truncation is shown in Table I for several system sizes. This method, referred to as Bound excitation Truncation (BT), is similar to that utilized by Poirier and co-workers; ${ }^{24,25}$ however, this scheme has different physical implications despite the underlying mathematics being the same. In Refs. 24 and 25, the underlying basis set used was that of harmonic oscillator eigenstates, which are known to have linear energy scaling, $E_{v}=\hbar \omega(v+1 / 2)$, as a function of the vibrational quantum number $v$ for oscillator frequency $\omega$. By contrast, the energy scaling of the free rotor is quadratic,

\begin{tabular}{|c|c|c|c|c|c|c|c|c|c|c|}
\hline \multirow[b]{2}{*}{$n$} & \multicolumn{2}{|r|}{$L=2$} & \multicolumn{2}{|c|}{$L=4$} & \multicolumn{2}{|c|}{$L=6$} & \multicolumn{2}{|c|}{$L=8$} & \multicolumn{2}{|c|}{$L=10$} \\
\hline & $N$ & Elements & $N$ & Elements & $N$ & Elements & $N$ & Elements & $N$ & Elements \\
\hline 2 & 6 & $2.3 \times 10^{1}$ & 19 & $1.1 \times 10^{2}$ & 44 & $3.3 \times 10^{2}$ & 85 & $7.1 \times 10^{2}$ & 146 & $1.3 \times 10^{3}$ \\
\hline 3 & 13 & $8.4 \times 10^{1}$ & 76 & $8.6 \times 10^{2}$ & 284 & $4.3 \times 10^{3}$ & 809 & $1.5 \times 10^{4}$ & 1925 & $3.9 \times 10^{4}$ \\
\hline 4 & 23 & $2.0 \times 10^{2}$ & 220 & $3.5 \times 10^{3}$ & 1236 & $2.8 \times 10^{4}$ & 4961 & $1.4 \times 10^{5}$ & 15847 & $5.1 \times 10^{5}$ \\
\hline 5 & 36 & $4.0 \times 10^{2}$ & 516 & $1.1 \times 10^{4}$ & 4116 & $1.2 \times 10^{5}$ & 22391 & $8.5 \times 10^{5}$ & 93392 & $4.2 \times 10^{6}$ \\
\hline 6 & 52 & $6.8 \times 10^{2}$ & 1048 & $2.6 \times 10^{4}$ & 11340 & $4.2 \times 10^{5}$ & 80907 & $3.8 \times 10^{6}$ & 430032 & $2.5 \times 10^{7}$ \\
\hline 7 & 71 & $1.1 \times 10^{3}$ & 1919 & $5.6 \times 10^{4}$ & 27161 & $1.2 \times 10^{6}$ & 247220 & $1.4 \times 10^{7}$ & 1639212 & $1.1 \times 10^{8}$ \\
\hline 8 & 93 & $1.6 \times 10^{3}$ & 3251 & $1.1 \times 10^{5}$ & 58447 & $2.9 \times 10^{6}$ & 663060 & $4.4 \times 10^{7}$ & 5383876 & $4.4 \times 10^{8}$ \\
\hline 9 & 118 & $2.3 \times 10^{3}$ & 5185 & $2.0 \times 10^{5}$ & 115600 & $6.5 \times 10^{6}$ & 1602688 & $1.2 \times 10^{8}$ & 15681136 & $1.5 \times 10^{9}$ \\
\hline 10 & 146 & $3.1 \times 10^{3}$ & 7881 & $3.3 \times 10^{5}$ & 213616 & $1.3 \times 10^{7}$ & 3559136 & $3.0 \times 10^{8}$ & 41376016 & $4.3 \times 10^{9}$ \\
\hline
\end{tabular}
$E_{l}=B l(l+1)$. This implies a different physical meaning between bounding of the two basis set indices. In the case of harmonic oscillators, the truncation parameter has a direct

TABLE I. Growth of basis size $N$ and number of non-zero Hamiltonian matrix elements with system size $n$ and truncation parameter $L$. 
relation to the energy of the system-i.e., polyad truncation is an energy-based criterion. Here, the truncation parameter can be viewed as an "occupation number," in that we have a bound number of rotor excitations that can be added to or removed from the system. This suggests a second quantization framework, similar to that of the Bose-Hubbard model. However, unlike the Bose-Hubbard model, the total number of excitations is bounded, not fixed ("=" becomes " $\leq$ "). It is therefore possible to think of the pinned rotor Hamiltonian under BT as a grand canonical ensemble of virtual particles. As a consequence of this, we are not able to easily make energy arguments in terms of the basis performance since we have the incorrect basis "scaling" with respect to the zeroth energy. For that we would need $\sum_{i=1}^{n} l_{i}^{2} \leq E$. Unfortunately, this makes the total basis size $(N)$ scaling intractable very quickly and is, therefore, not utilized. For proper exploration of the subtle implications of this difference- $l$ vs $l^{2}-$ a full phase space analysis should be employed, but that is far beyond the scope of what is presented here. With regards to $N$ and its scaling, it should also be noted that $N$ was greatly reduced by the utilization of symmetry. Specifically, if we let $\sum_{i=1}^{n} l_{i}=l_{\text {тот }}$ and $\sum_{i=1}^{n} m_{i}=m_{\text {тот }}$, then the two symmetries in question are the even and odd parity of $l_{\text {тот }}$ and the integer value of $m_{\text {тот }}$. Both the states we wish to compute come from the same $m_{\text {тот }}=0$ block; however, $|0\rangle$ has even $l_{\text {тот }}$ character, whereas $|1\rangle$ has odd $l_{\text {тот }}$ character.

For comparison to the many-body (MB) method described above, we also utilize two approximate approaches. Currently, neither of the methods yield eigenfunctions, only eigenenergies, and are, therefore, not relevant in the latter conversation about entanglement. However, these approximations do provide qualitative insight, and, at the very least, yield a criterion for properly choosing $L$-values since they can easily give us asymptotic behavior. The first approximate method we used is second order perturbation theory (PT). It turns out that the second order correction to the ground state energy is analytic since the dipole potential can be expressed in terms of coordinate operators, $\hat{x}_{i}, \hat{y}_{i}$, and $\hat{z}_{i}$. These operators produce very strict selection rules, and as a result, there are only two nonzero matrix elements (technically three, but two have the same value due to exchange symmetry),

$$
\begin{aligned}
E_{0}^{\mathrm{PT}}(n)= & -\frac{\beta^{2}}{4 \alpha}\left(\left|\left\langle 1010\left|\hat{V}_{12}\right| 0000\right\rangle\right|^{2}\right. \\
& \left.+2\left|\left\langle 111-1\left|\hat{V}_{12}\right| 0000\right\rangle\right|^{2}\right) \sum_{i=1}^{n-1} \frac{n-i}{i^{6}} \\
= & -\frac{\beta^{2}}{6 \alpha} \sum_{i=1}^{n-1} \frac{n-i}{i^{6}} .
\end{aligned}
$$

This yields a simple expression and provides a baseline for comparison. In the low coupling regime, this approximation works well, in that it at least captures the qualitative behavior; of course, it becomes less useful in the high coupling limit, but that is a well-known limitation of PT.

The second approximation is a pairwise additive approach (PW)-i.e., we assume $E_{0}$ for the total system is simply the sum of pair energies. The PW energies are computed by diagonalizing the Hamiltonian for a single pair $(n=2)$ of rotors that are separated by an integer multiple of the lattice spacing. This yields

$$
E_{0}^{\mathrm{pw}}(n)=\sum_{i=1}^{n-1}(n-i) E_{0}^{i}
$$

Whether exact or approximate, eigenenergy calculation was not our primary goal. In fact, most of the tools developed above are only used as a proof for the accuracy the MB eigenstates, which are needed to compute $S_{A}$. To begin, we first partition the total system into two subsystems, $A$ and $B$. In general, this partitioning can be arbitrary, but for computational simplicity, it useful to choose a division that is respected by the underlying basis being used. Since we utilize a direct product of single particle basis functions, it stands to reason that a single particle partitioning (SPP) would be an obvious choice. That is to say, we should choose $A$ to be a single rotor and $B$ to be the rest in the chain. This type of partitioning will yield a measure of how an individual rotor is entangled to the full system-i.e., how much information is gained about the system by doing a measurement on a single member. In practice though, doing an experimental measurement on a single rotor would be highly non-trivial since the lattice spacings necessary for reasonable entanglement would be on the order of the wavelength of the probe, e.g., a far-infrared photon.

In any event, we wish to compute the von Neumann entropy for the subsystem $A$,

$$
S_{A}=-\sum_{i=1}^{M} \lambda_{i}^{A} \ln \lambda_{i}^{A},
$$

where $\left\{\lambda_{1}^{A}, \lambda_{2}^{A}, \ldots, \lambda_{M}^{A}\right\}$ is the set of eigenvalues of the reduced density operator,

$$
\hat{\rho}_{A}=\operatorname{Tr}_{B}[\hat{\rho}]
$$

To compute the eigenvalues, we expand $\hat{\rho}_{A}$ in the basis of the subsystem $A$ and diagonalize. In the context of SPP where $A$ is the $j$ th rotor and $B$ is the rest, we can recast $\hat{\rho}_{A}$ by exploiting the aforementioned basis structure,

$$
\left\langle l_{j}^{\prime} m_{j}^{\prime}\left|\hat{\rho}_{j}^{k}\right| l_{j} m_{j}\right\rangle=\sum_{\mathbf{n}} c_{l_{j}^{\prime} m_{j}^{\prime} \mathbf{n}}^{k} c_{l_{j} m_{j} \mathbf{n}}^{k *},
$$

where $c_{l_{j} m_{j} \mathbf{n}}^{k}$ are the expansion coefficients of wavefunction $|k\rangle$ and $\mathbf{n}=\left\{l_{1} m_{1}, \ldots, l_{j-1} m_{j-1}, l_{j+1}, m_{j+1}, \ldots, l_{n} m_{n}\right\}$ is the configuration over which the tracing occurs. For SPP, the basis size of the represented reduced density matrix is no larger than that of a single particle Hilbert space-i.e., the set $\{|l m\rangle\}_{l=0}^{L}$, which makes diagonalization trivial.

\section{RESULTS}

For rotor-doped NMAs to be viable, the rotor-rotor interactions must be long range enough as to still be significant at realistic lattice distances. For linear assemblies of $\mathrm{C}_{60}$ cages, it has been shown that the center-to-center distance is 19.0 bohrs. ${ }^{17} \mathrm{HF}$ represents a strong candidate molecule since the dipole strength is still significant, even at several times that distance; however, one cannot simply ignore cage interactions. To properly describe a basic NMA, perhaps comprised of several HF@ $\mathrm{C}_{60}$ cages confined to a linear configuration inside a 
TABLE II. Hamiltonian parameters (in $\mathrm{cm}^{-1}$ ) for the screened and unscreened dipole moment of HF.

\begin{tabular}{lcc}
\hline \hline & Screened & Unscreened \\
\hline$\alpha$ & 20.561 & 20.561 \\
$\beta$ & 1.0 & 16.5 \\
$\beta / \alpha$ & 0.049 & 0.802 \\
\hline \hline
\end{tabular}

single walled carbon nanotube (SWNT), the effects of the cages on the dipole-dipole interactions must be taken into account. Recent studies have shown that the rotor-rotor interaction can be approximated by treating the cage effects as a screening of the dipole moment. ${ }^{16,17}$ This provides a good foundation to treat a basic rotor-doped NMA as a chain of pinned dipoles, described by Eq. (1). For HF, the gas phase dipole moment is $1.8265 \mathrm{D},{ }^{26}$ while for $\mathrm{HF} @ \mathrm{C}_{60}$, this value is reduced to $0.45 \mathrm{D} .{ }^{16}$ The value of $\alpha=20.561 \mathrm{~cm}^{-1}$ was used for both cases. ${ }^{27}$

\section{A. Screened hydrogen fluoride: Low coupling}

Hamiltonian parameters for the screened HF system can be seen in Table II. With the screening, this system represents the low end of the coupling regime-i.e., $\beta / \alpha \ll 1$. However, due to advances in fullerene chemistry, one could imagine that these devices could become highly tunable, allowing for an arbitrary combination of parameters. In any event, this configuration, screened HF, represents the most physically realistic device that could be made using current methods and is, therefore, an important staging point for future understanding.

\section{Energies}

With this in mind, we computed $E_{0}$ for NMA chains of screened HF with $n=2-10$ using the three methods discussed in Sec. II. For the MB Hamiltonian, we computed the eigenspectrum for $L=\{2,4,6,8,10\}$. These results are given in Table III. In this low coupling regime, we see that $E_{0}$ is converged for all calculations beyond $L=2$. Moreover, since the coupling is small, the two approximate methods yield results within $10^{-3} \mathrm{~cm}^{-1}$ of the exact results. Looking deeper, we can conclude that since all of the exact results are converged beyond $L=2, L=4$ is the "appropriate level of theory" for all relevant systems sizes. This is promising since the basis size $(N)$ needed for the largest system size $n=10$ is only $N=7881$, which is a trivial diagonalization. This is dramatic savings over $L=10$, which yields a basis size of $N=41376$ 016. In practice, the latter calculation is done using sparse iterative methods, reducing it to a somewhat trivial calculation by today's standards. ${ }^{28}$ In any event, the computed values are numerically identical, obviating the latter calculation, and, more importantly, the need for atypical computational resources.

A subtle issue that is difficult to glean from Table III is a lack of "size extensivity," a well-known problem in the field of quantum chemistry. ${ }^{29}$ We can see that for $L=2$, we get results that are within $\sim 10^{-6}$ to $\sim 10^{-4} \mathrm{~cm}^{-1}$ from $L=4$, which is $<0.1 \%$ difference. However, this does not tell the entire story. If we compute the chemical potential, $\mu=E_{0}^{n}-E_{0}^{n-1}$, it becomes obvious that as we tend toward large $n, L=2$ yields the wrong asymptotic behavior. This can be observed in the top panel of Fig. 2. At large $n$, the addition of a new rotor should have almost no effect on the total energy of the system-i.e., $\mu$ should tend toward a constant value. That is not the case for $L=2$, and we must increase to $L=4$ to get the desired result. Felker and Bačić observed the same large size behavior in their recent work. ${ }^{30}$ Upon analysis of their computed ground state energies for assemblies of $\mathrm{H}_{2} \mathrm{O} @ \mathrm{C}_{60}$, we see $\mu$ displays the same constant behavior with increasing $n$. This is true despite their Hamiltonian including translational motion of the dopant molecule. From all this, we can conclude that it is not enough to rely only on the numerical convergence of the eigenenergies; in fact, doing so could yield the wrong bulk behavior.

\section{Entanglement}

Using Eq. (11), we computed $S_{A}(n)$ for both $L=2$ and $L=4$ with the SPP of $j=1$. These results can be seen in Fig. 3 (top panel). Again, the size extensivity issue is apparent for $L=2$, exhibited by $S_{A}$ decreasing with increased $n$. Also as before, we regain the proper bulk behavior by increasing to $L=4$. To explain this behavior, we examine the configuration entropy of the basis, $S_{c}=\ln N$, which is determined by the number of accessible states. If we do not implement BT and simply use a standard direct product (DP) basis, with $d$ single

TABLE III. Ground state energy (in $10^{-3} \mathrm{~cm}^{-1}$ ) for screened HF chains computed using three methods: $\mathrm{MB}, \mathrm{PW}$, and PT. The MB energies, ${ }^{L} E_{0}^{\mathrm{MB}}$, were found at various values of $L$ to show convergence. The last two columns (italicized) show the results of perturbation theory and of the pairwise approximation.

\begin{tabular}{lccccccc}
\hline \hline$n$ & ${ }^{2} E_{0}^{\mathrm{MB}}$ & ${ }^{4} E_{0}^{\mathrm{MB}}$ & ${ }^{6} E_{0}^{\mathrm{MB}}$ & ${ }^{8} E_{0}^{\mathrm{MB}}$ & ${ }^{10} E_{0}^{\mathrm{MB}}$ & $E_{0}^{\mathrm{PT}}$ & $E_{0}^{\mathrm{PW}}$ \\
\hline 2 & -8.10537 & -8.10557 & -8.10557 & -8.10557 & -8.10557 & -8.10596 & -8.10557 \\
3 & -16.3622 & -16.3635 & -16.3635 & -16.3635 & -16.3635 & -16.3385 & -16.3481 \\
4 & -24.6308 & -24.6350 & -24.6350 & -24.6350 & -24.6350 & -24.5823 & -24.6018 \\
5 & -32.9000 & -32.9088 & -32.9088 & -32.9088 & -32.9088 & -32.8280 & -32.8575 \\
6 & -41.1682 & -41.1832 & -41.1832 & -41.1832 & -41.1832 & -41.0742 & -41.1138 \\
7 & -49.4349 & -49.4577 & -49.4577 & -49.4577 & -49.4577 & -49.3206 & -49.3702 \\
8 & -57.7000 & -57.7324 & -57.7324 & -57.7324 & -57.7324 & -57.5671 & -57.6267 \\
9 & -65.9635 & -66.0071 & -66.0071 & -66.0071 & -66.0071 & -65.8136 & -65.8832 \\
10 & -74.2253 & -74.2818 & -74.2818 & -74.2818 & -74.2818 & -74.0601 & -74.1398 \\
\hline \hline
\end{tabular}



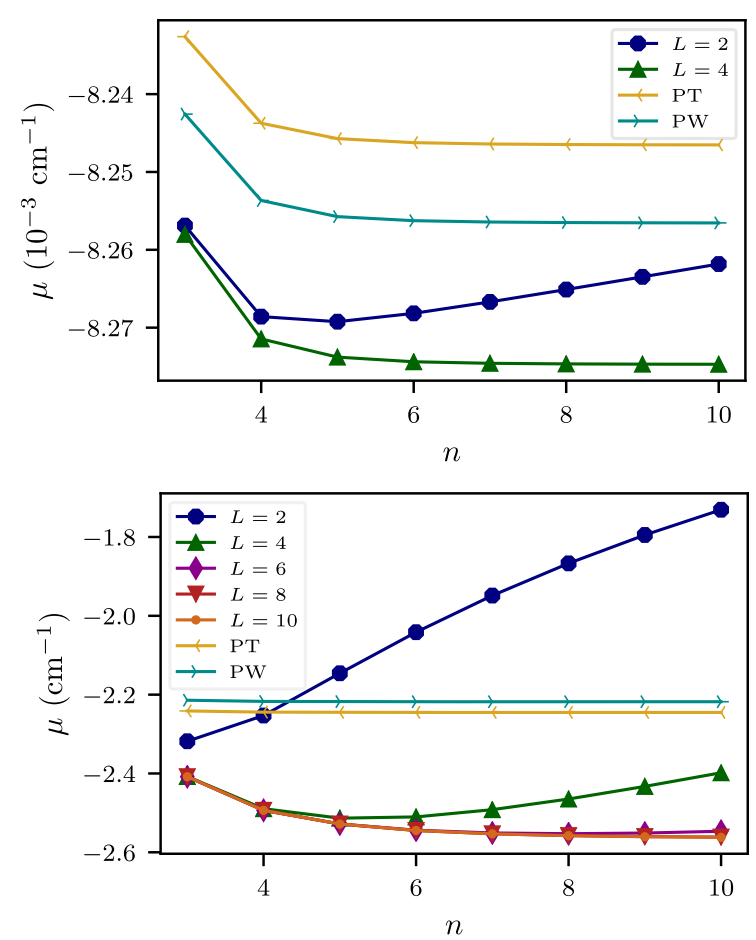

FIG. 2. Chemical potential as a function of $n$ for the ground state of screened (top panel) and unscreened (bottom panel) HF. Different $L$ truncation values are used, along with the chemical potential computed using the pairwise (PW) and perturbative (PT) approximations.

particle states, then $N$ would scale exponentially with the number of rotors, $N^{\mathrm{DP}}=d^{n}$. This would yield $S_{c}$ as a linear function of $n, S_{c}^{\mathrm{DP}}(n)=n \ln d$, which is extensive. However, with BT, $N$ scales as a power law in $n, N^{\mathrm{BT}}(n) \propto n^{L}$, yielding $S_{c}^{\mathrm{BT}}(n)=L \ln n+O(1)$, which is fundamentally different
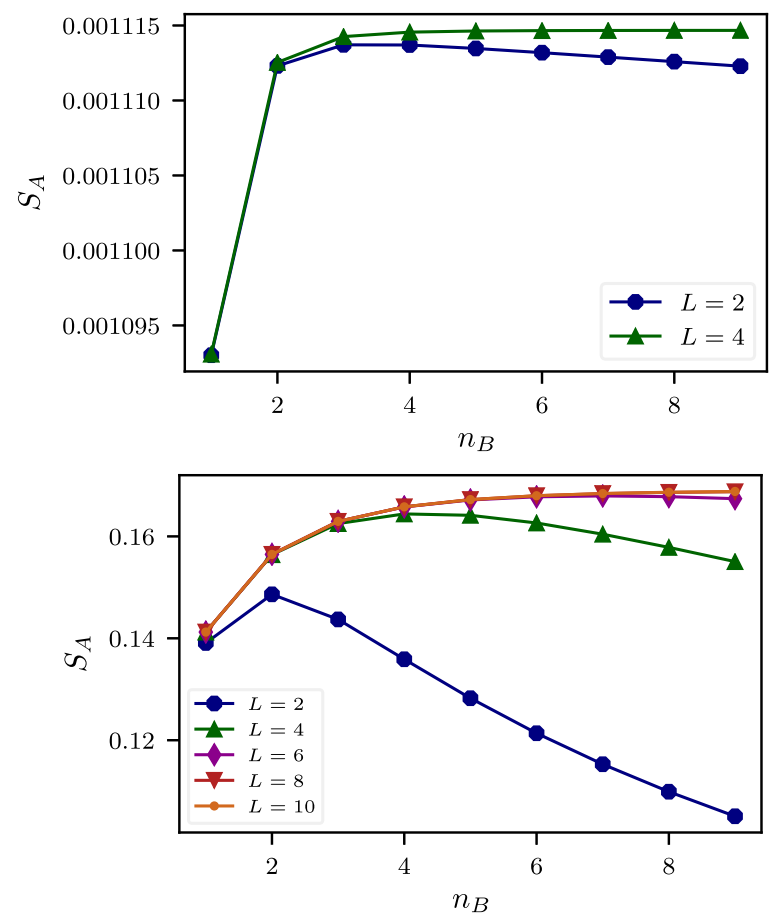

FIG. 3. von Neumann entropy as a function of the size $n_{B}$ of partition $B$ for $j=1$ partitioning for the ground state of screened (top panel) and unscreened (bottom panel) HF for different $L$ truncation values. scaling than DP. This suggests the heuristic argument that when the configuration entropy is non-extensive, the basis may not grow sufficiently quickly to faithfully represent the eigenstates of the Hamiltonian.

Thus, $L$ should have some functional dependence on $n$ to ensure proper large $n$ behavior-i.e., a constant $L$ will eventually fail. From the computed energies, we can conclude that $L=4$ is sufficient for the $n$ range studied here since it yields the same results as larger $L$ values. However, we will see later that at larger coupling, $L=4$ breaks down in the same manner, implying that $L$ is not only dependent on $n$ but also on the coupling strength. Beyond this, not much can be said about the exact form of $L$. A full analysis of the parameter space would be necessary to define a proper $L$-function, and this is left for future research.

\section{Correlations}

With unfettered access to numerically exact wavefunctions, one could conceivably analyze any property of the system to explore entanglement. The most obvious is, of course, entanglement entropy; however, another quantity that holds interest is the dipole correlation operator, $C_{\alpha_{i} \alpha_{j}}$. If we let $\mathbf{e}_{i}=e_{x_{i}} \mathbf{i}+e_{y_{i}} \mathbf{j}+e_{z_{i}} \mathbf{k}$ be the unit vector representing the orientation of the $i$ th dipole, then we can define

$$
C_{\alpha_{i} \alpha_{j}}=\left\langle 0\left|\mathrm{e}_{\alpha_{i}} \mathrm{e}_{\alpha_{j}}\right| 0\right\rangle,
$$

where $\mathrm{e}_{\alpha_{i}}$ is the Cartesian component $\alpha=x, y, z$.

Using this, we computed the $x$ and $z$ correlation functions between $i=6$ and $j=1-10$. This can be seen in the top panel of Fig. 4. Due to symmetry, the $x$ and $y$ functions have the same value, and, therefore, the $y$ analysis is omitted. Both $x$ and $z$ have a maximum value of $\sim 1 / 3$ at $j=6$, which for low coupling makes sense, since the total dipole autocorrelation is unity and the ground state is nearly isotropic. What is more interesting is that in this coupling regime, we see only small nearest neighbor correlations, with effectively zero interactions beyond, for both $x$ and $z$. However, the $z$ projection (the principal axis of the chain) has positive correlation, whereas $x$ is negative. This difference in sign can be understood by referring to Eq. (2), where we see that the $x$ and $z$ contributions to the potential energy have opposite sign: $x$ is positive and $z$ is negative. Moreover, we know the ground state wavefunction is expected to have greatest amplitude in regions of more negative potential energy. This implies that the correlations will be maximal when $z_{i}$ and $z_{j}$ have the same sign since that corresponds to the most negative value of the potential. Conversely, $x_{i}$ and $x_{j}$ must have opposite signs for their contributions to the potential to maximally negative. Despite all of this, the main conclusion these results show is that a screened HF molecule in the current peapod geometry is nearly uncorrelated, especially beyond the nearest neighbor. However, the situation will be quite different in the case of unscreened HF (vide infra).

\section{B. Unscreened hydrogen fluoride: Large coupling}

Although the screened HF NMA represents the most physically realistic system, we have seen in Sec. III A that the 

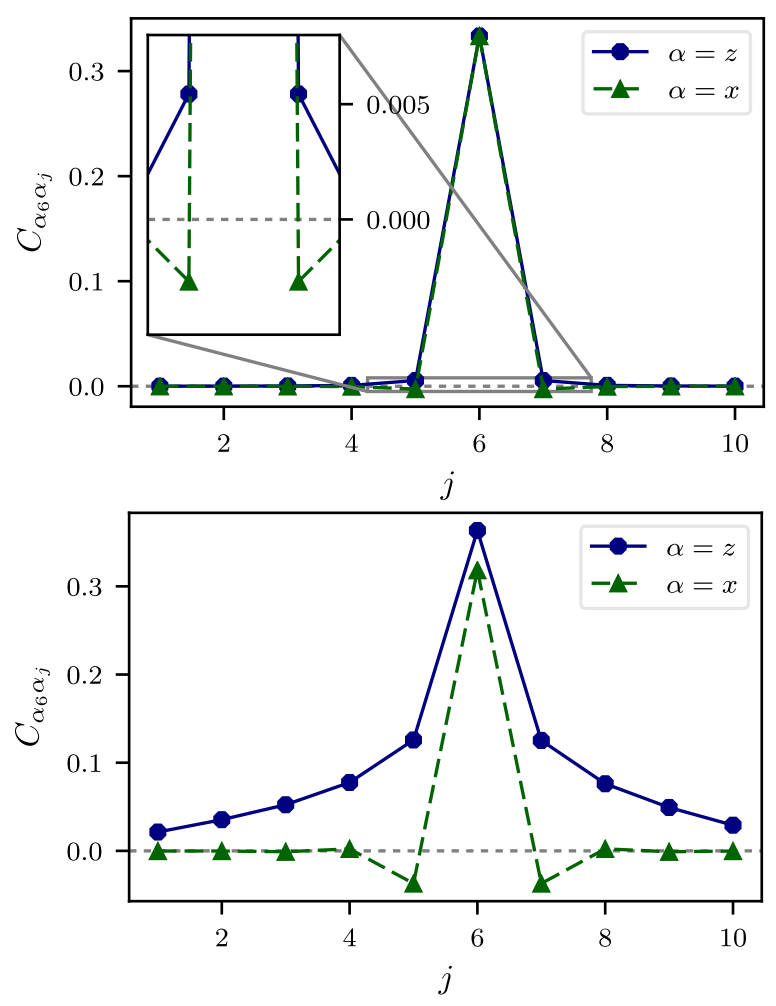

FIG. 4. Ground state correlation functions between site 6 and site $j$ for screened (top panel) and unscreened (bottom panel) HF for $n=10$.

features of the entanglement and correlations are somewhat uninteresting. This is due to the reduced level of interaction resulting from the dipole screening; however, one could envision doping the buckypeapod with a new molecule of larger dipole moment or smaller rotational constant (e.g., LiF) which would still maintain large coupling even in the presence of screening. In any event, unscreened HF represents an NMA with large coupling and is, therefore, of significance in terms of entanglement analysis.

\section{Energies}

Similar to the screened case in Sec. III A, we computed $E_{0}$ for $n=2-10$ with $L=\{2,4,6,8,10\}$. These results can be seen in Table IV. Here, we see a stark contrast to Table III. The values do not converge quickly, and the approximations are much worse. For PT, this is to be expected since the larger perturbation makes the underlying basis less appropriate. In fact, in the case of $\beta / \alpha \gg 1$, the spherical harmonics would be the incorrect choice, and one should utilize the eigenstates of the potential (the position representation) since the potential would be the dominant term. As for PW, the longer range coupling due to increased dipole strength makes a purely additive approximation incorrect-i.e., the larger coupling makes $E_{0}$ for the total system less like a sum of single particle energies. In any event, none of these methods yield results that would be considered horrendous; however, some would not be considered spectroscopic. The smallest basis, $L=2$, yields decent results (within 1\%) for $n=2$, but we must increase to $L=6$ to get $<1 \%$ convergence for $n=10$. As we now know, though, convergence analysis does not tell the entire story. The more fundamental issue can be observed in Fig. 2 (bottom panel), where we see $\mu$ for the unscreened system. There, the size extensivity issue (discussed above) is much more pronounced than it was for screened dipoles. In fact, for $L=2$, we see completely incorrect behavior. Even for $L=4$, the results break down quickly. One thing of note is that both approximate methods, despite being relatively inaccurate, display the proper asymptotic scaling. Moreover, we can glean from Fig. 2 that we must choose at least $L=6$ for our results to be extensive. Even that is somewhat suspect since it is clear that $n=10$ is very close to the limit for $L=6$. To go beyond this, $L=6$ would no longer be sufficient, but if the goal is to get spectroscopic accuracy with the least amount of computational effort, then $L=6$ is the best choice.

\section{Entanglement}

This behavior is echoed when we look at $S_{A}$. In Fig. 3 (bottom panel), we see that $L=2$ and $L=4$ results are blatantly incorrect, in that they have very wrong asymptotic behavior. Moreover, $L=6$ is beginning to turn around. As was laid out before, this can be somewhat understood by the fact that the configuration entropy has the wrong scaling with $n$. Also, it can now clearly be seen that for proper scaling to be maintained throughout (given the constraint of minimal computational effort), one would need to derive an explicit expression for the $n$ dependence of $L$.

Since the behavior of $S_{A}$ is a little more interesting in this case, we can also look at how it changes with site index. In the top panel of Fig. 5, we see $S_{A}$ as a function of $j$ for

TABLE IV. Ground state energy (in $\mathrm{cm}^{-1}$ ) for unscreened HF chains computed using three methods: MB, PW, and PT. The MB calculations were carried out at various values of $L$ to show convergence.

\begin{tabular}{lccccccc}
\hline \hline$n$ & ${ }^{2} E_{0}^{\mathrm{MB}}$ & ${ }^{4} E_{0}^{\mathrm{MB}}$ & ${ }^{6} E_{0}^{\mathrm{MB}}$ & ${ }^{8} E_{0}^{\mathrm{MB}}$ & ${ }^{10} E_{0}^{\mathrm{MB}}$ & $E_{0}^{\mathrm{PT}}$ & $E_{0}^{\mathrm{PW}}$ \\
\hline 2 & -2.16527 & -2.17940 & -2.17941 & -2.17941 & -2.17941 & -2.20684 & -2.17941 \\
3 & -4.48352 & -4.58712 & -4.58764 & -4.58764 & -4.58764 & -4.44817 & -4.39356 \\
4 & -6.73642 & -7.07644 & -7.08112 & -7.08114 & -7.08114 & -6.69253 & -6.61074 \\
5 & -8.88218 & -9.58981 & -9.60920 & -9.60939 & -9.60940 & -8.93743 & -8.82846 \\
6 & -10.9237 & -12.1000 & -12.1531 & -12.1540 & -12.1540 & -11.1824 & -11.0463 \\
7 & -12.8723 & -14.5918 & -14.7040 & -14.7071 & -14.7072 & -13.4275 & -13.2642 \\
8 & -14.7392 & -17.0565 & -17.2568 & -17.2648 & -17.2650 & -15.6726 & -15.4821 \\
9 & -16.5341 & -19.4893 & -19.8079 & -19.8250 & -19.8255 & -17.9177 & -17.7001 \\
10 & -18.2650 & -21.8875 & -22.3545 & -22.3864 & -22.3876 & -20.1628 & -19.9180 \\
\hline \hline
\end{tabular}



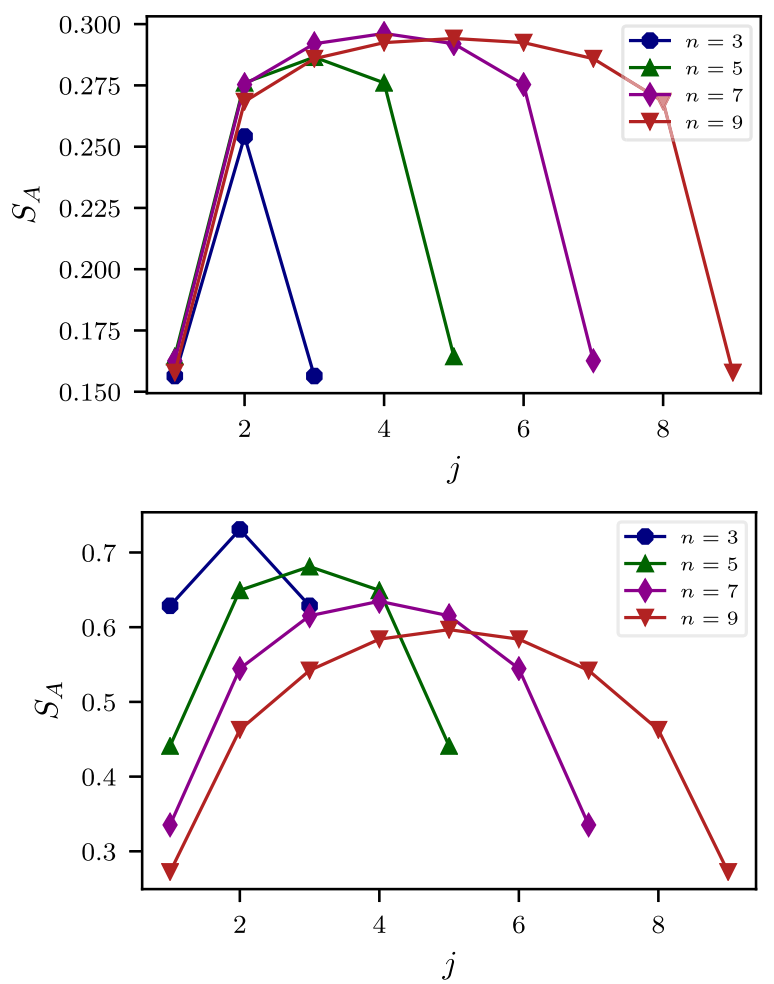

FIG. 5. von Neumann entropy as a function of SPP site location for the ground (top panel) and first excited state (bottom panel) of unscreened HF, computed with $L=5$.

$n=\{3,5,7,9\}$. This plot yields insight into how entangled a rotor is to the rest of the system. We see right away that the end rotors have very different properties than those in the middle of the chain-i.e., they are much less entangled to the rest of the system than the inner rotors. This is true regardless of $n$. As $n$ gets larger, however, the level of entanglement of the inner rotors becomes more smooth, implying a constant $S_{A}$ value at very large $n$. The fact that the endpoints exhibit such different behavior compared to the middle could be useful, depending on the desired application.

\section{Correlations}

As before, we computed $C_{\alpha_{i} \alpha_{j}}$. This can be seen in the bottom panel of Fig. 4. One major difference between the screened and unscreened system is that now the $z$ autocorrelation is much larger than the isotropic value of $1 / 3$, implying that the fluctuations of the orientation about the average are smaller. This is to be expected since in the infinite coupling limit the system would become ferroelectric. We can also note that the $z$ correlation has a much larger range. In fact, at this system size, every rotor is correlated with those in the middle $\left(C_{z_{6} z_{j}}\right.$ is not zero at the endpoints). For the $x$ component, we see a more exaggerated anti-correlation with nearest neighbors than for the screened system.

\section{Excited states}

Along with the preceding analysis of $|0\rangle$, we also analyzed the entanglement behavior of $|1\rangle$. Here, we define $|1\rangle$ to be the ground state of the $m_{\mathrm{tot}}=0$ and $l_{\text {tot }}$ odd symmetry block. Energetically, this is the second state in the total (without symmetry) eigenspectrum. This state is also of interest since it is E1 allowed-i.e., the transition dipole moment, $\left|\frac{1}{n}\left\langle 1\left|\sum_{i=1}^{n} \hat{\mathbf{e}}_{i}\right| 0\right\rangle\right|^{2}$, is non-zero.

Like before, we computed $S_{A}$ via Eq. (11). Figure 6 shows the results of this for the $j=1$ SPP as a function of $n$ for various values of $L$ for the screened (top panel) and unscreened (bottom panel) cases. For the screened system, all values of $L$ yield converged results. However for the unscreened system, it is clear that $L=1$ is not sufficient, vis-à-vis the large numerical discrepancy between $L=1$ and $L=3$, even at small $n$. The $L=5$ results are taken to be converged since the $L=6$ results were converged for $|0\rangle$. In any event, we are not necessarily interested in a full convergence study of $|1\rangle$. We are more interested in the qualitative behavior of $S_{A}$. In particular, how it is different from $|0\rangle$. This main difference is that $S_{A}$ decreases with increased $n$-i.e., the amount by which each rotor is entangled to the rest of the system decreases as the system size is increased. This behavior is the exact opposite of the ground state behavior and is somewhat counter intuitive. Upon further analysis, though, this behavior does make sense. If we assume $|1\rangle$ can be approximated as a symmetric combination $|\operatorname{sym}(n)\rangle$ of a single $l$ excitation, then we can explain the decrease in $S_{A}$ by noting that as $n$ increases, the amount of information gained by measuring the $j=1$ rotor decreases. To be more explicit, start with $n=2$. This would yield an approximate excited state of the form $|\operatorname{sym}(2)\rangle=\frac{1}{\sqrt{2}}(|1000\rangle+|0010\rangle)$. A measurement of $l$ for $j=1$ would either yield zero or one. However, it would also yield perfect information about $j=2$ since the system is in a Bell state and has maximal
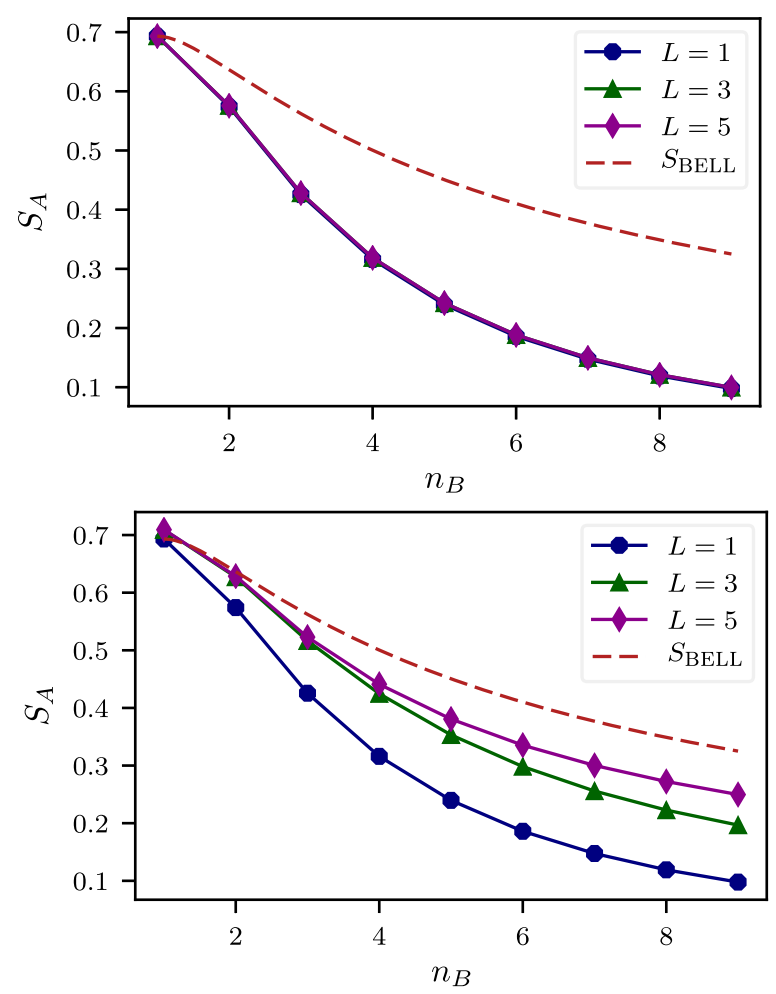

FIG. 6. von Neumann entropy as a function of chain length for $j=1$ partitioning with $L=1,3,5$ for the first excited state of screened (top panel) and unscreened (bottom panel) HF. 
entanglement entropy $\left(S_{A}=\ln 2\right)$ in the $\{|00\rangle,|10\rangle\}$ subspace. Now, if we increase the system size to $n=3$, then $|1\rangle$ would have the form $|\operatorname{sym}(3)\rangle=\frac{1}{\sqrt{3}}(|100000\rangle+|001000\rangle+|000010\rangle)$. Again, measuring $l$ for $j=1$ would yield either one or zero, but this time we gain less information about the rest of the system. If we measure $l=1$, then $B$ is in the state $|0000\rangle$; however, if $l=0$ then $B$ is either in $|1000\rangle$ or $|0010\rangle$. Extending this analysis to arbitrary $n$, we can derive a general expression for the "Bell state entropy" as a function of $n, S_{\mathrm{BELL}}=\ln (n)-\frac{n-1}{n} \ln (n-1)$, which has an asymptotic limit of zero. This result can also be seen in Fig. 6. Furthermore, the overall decrease in $S_{A}$ with increased $n$ can be seen in the bottom panel of Fig. 5. There we see similar behavior to that of $|0\rangle$, in that both exhibit the same "rainbow" pattern, but now the overall rainbow shifts downward with $n$.

Lastly, we also computed $S_{A}$ for the superposition state, $|\psi\rangle=\gamma|0\rangle+\sqrt{1-\gamma^{2}}|1\rangle$, where $\gamma$ is a real-valued parameter, and $|0\rangle$ and $|1\rangle$ are the aforementioned ground and excited states for the HF chain. On its own, this state can be viewed as a two -level qubit living on the prime meridian of the Bloch sphere. Whether or not this state would constitute a viable
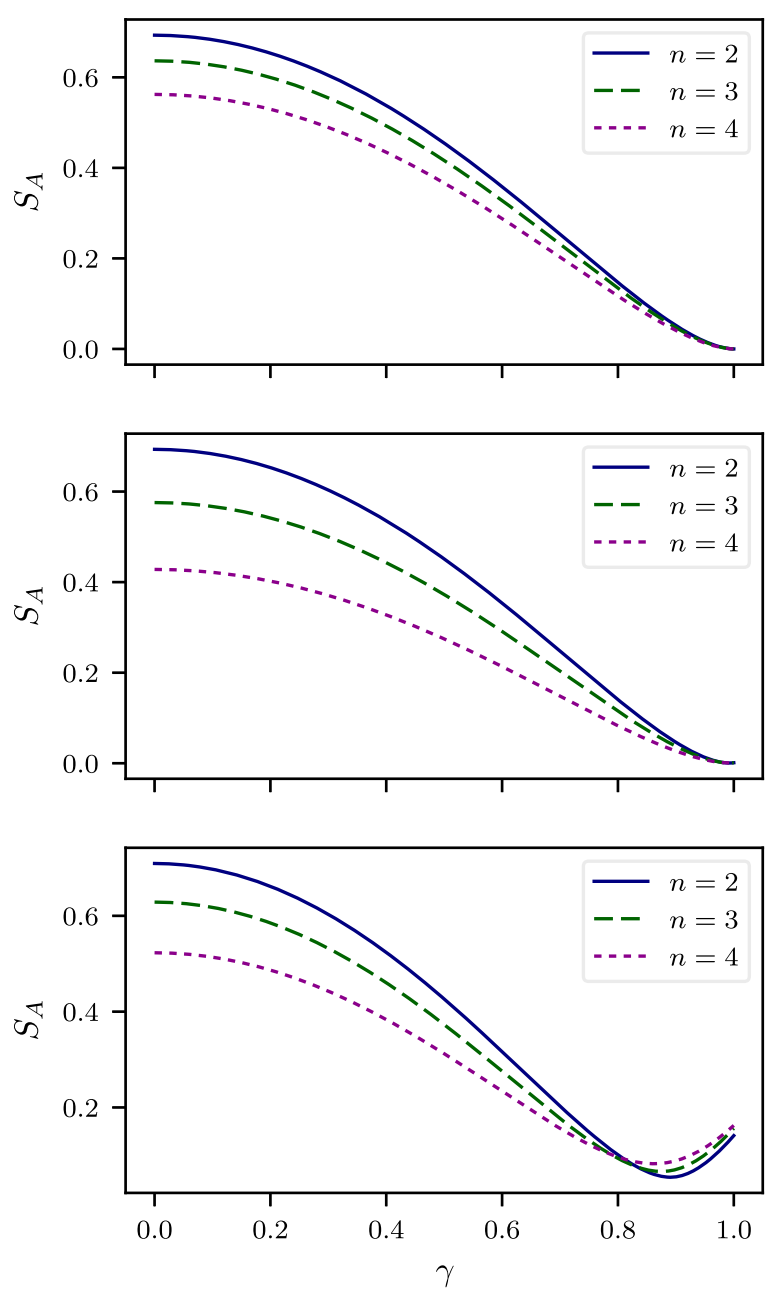

FIG. 7. von Neumann entropy as a function of state coefficient, $\gamma$, for a superposition of the ground and first excited states of HF, computed with $L=5$. Free rotor (top panel), screened (middle panel), and unscreened (bottom panel) dipole results are shown. In the free rotor case, the first excited level is degenerate, but we are interested in the $\beta \rightarrow 0$ limit, so we choose $|1\rangle=|\operatorname{sym}(n)\rangle$. candidate for quantum computation is beyond the scope of what is presented here, but one could imagine applying some operation on the system as to induce this state with a tunable $\gamma$. Results for screened, unscreened, and free dipoles are shown in Fig. 7, which shows $S_{A}(\gamma)$ for the $j=1$ SPP. Predictably, the entanglement is maximum when $\gamma=0$ for all three systems, which corresponds to a pure $|1\rangle$ state. For both the free rotor and screened dipole cases, $S_{A}$ decreases monotonically as $\gamma$ goes to unity. The free rotor ground state is a product state, resulting in $S_{A} \rightarrow 0$ as expected. The behavior of the screened dipole ground state is similar to that of the free rotor since the entanglement is low. However for the unscreened system, $|0\rangle$ is not a product state and therefore has a non-vanishing entanglement entropy.

\section{CONCLUDING REMARKS}

We have studied quantum entanglement in a chain of rotors with dipole interactions using a variational description. To do so, we have developed a basis truncation scheme that allows the treatment of systems with a varying number of rotors. We have tested the approach for a chain of $\mathrm{HF} @ \mathrm{C}_{60}$ molecules. Both the weakly (screened dipoles) and strongly (unscreened dipoles) interacting regimes were considered. We have shown that to maintain the so-called size extensivity of the calculation, the $L$ truncation parameter has to scale with the size of the system. The loss of size extensivity was more pronounced for the unscreened case as expected. Our results show that screened HF leads to very short correlations and therefore minimal entanglement while its unscreened counterpart exhibits correlations that extend much farther with much higher particle entanglement.

The quest for reliable, inexpensive QIDs has been ongoing for many years, and much progress has made. The introduction of endofullerenes - i.e., the confinement of a molecule inside a closed fullerene structure-has ushered in a new era of NMA research. The quantification of entanglement in endohedral fullerenes is a first step in the exploration of their adequacy as QID candidates. In this respect, it is important to note that quantum computation schemes have already been explored in the context of trapped cold polar molecules, ${ }^{31-34}$ where the rotational and dipolar coupling terms of the Hamiltonian are identical to the terms in Eq. (1). Beyond this, Refs. 19-22 offer very interesting insights into direct applications of polar molecule assemblies for quantum computing. To that end, they treat these assemblies as collections of qubits and then study register initialization, feasibility of the entangling CNOT operation, and effects of temperature. Future work will focus on reconciling our use of the full Hilbert space of continuous rotors with that of the qubit representation.

From the present results, we were able to compute and characterize several important quantities in terms of quantum information, shedding light on several possible levels of tunability. Moreover, we also demonstrated that QID devices of this type live in the realm of realistic possibility. A rotor-doped buckypeapod of HF could be realized with current experimental techniques and under the screened dipole approximation does exhibit a small amount of quantum entanglement. If HF is changed to an appropriate molecule, we showed that the 
entanglement behavior is much more pronounced. The "desired" amount of entanglement will depend on the proposed quantum information scheme.

To truly understand the depth and breadth of this model, a full analysis of the parametric state space will be necessary. This could unlock interesting regimes, which could establish parameter settings for desired device effects-i.e., we could find values of $\alpha$ and $\beta$ that we would want to target. For instance, recent quantum Monte Carlo calculations of dipolar rotor chains suggest a transition from a disordered to an ordered phase near $\alpha \approx \beta{ }^{35,36}$ Note that the latter study did not address the issue of entanglement. Such a calculation would require a Monte Carlo estimator of the entanglement entropy such as the replica trick recently implemented for continuous space. $^{37-40}$

We conclude by observing that the present approach can be applied to non-linear molecules by expansion of the model to generic, asymmetric tops, while the inclusion of translational motion coupled to the rotational motion would serve to make the model more realistic. ${ }^{30}$ In this context, one would require a potential energy surface for the $\mathrm{HF} @ \mathrm{C}_{60}$ interaction. Such a potential has recently been published by our group. ${ }^{41}$ One could also envisage more elaborate two- or threedimensional lattice geometries that could obviate the need for polyatomic dopants. Moreover, external electric fields with either spatial (e.g., non-uniform transverse fields) or temporal (e.g., pulses) variation could also be utilized to modulate the entanglement properties. The analysis presented here only looked at single particle partitioning, but the methods can easily be extended to subsystems with more than a single particle. Complicated, multi-dimensional geometric configurations would open the door for a wide variety system partitionings. One could imagine a "sheet" of rotors, where some area of the sheet defines the subset $A$, or a cube, where $A$ is some internal volume. The latter could allow us to study fundamental questions such as the area law scaling of entanglement entropy. ${ }^{40,42-45}$

\section{ACKNOWLEDGMENTS}

This research has been supported by the Natural Sciences and Engineering Research Council of Canada (NSERC), the Ontario Ministry of Research and Innovation (MRI), the Canada Research Chair program, and the Canada Foundation for Innovation (CFI) and the Canada First Research Excellence Fund (CFREF). We thank M. Nooijen and C. Herdman for useful discussions.
${ }^{1}$ G. E. Moore, Proc. IEEE 86, 82 (1998).

${ }^{2}$ C. H. Bennett and D. P. DiVincenzo, Nature 404, 247 (2000).

${ }^{3}$ M. D. Lukin, M. Fleischhauer, R. Cote, L. M. Duan, D. Jaksch, J. I. Cirac, and P. Zoller, Phys. Rev. Lett. 87, 037901 (2001).

${ }^{4}$ R. H. Hadfield, Nat. Photonics 3, 696 (2009).

${ }^{5}$ H. W. Kroto, A. W. Allaf, and S. P. Balm, Chem. Rev. 91, 1213 (1991).

${ }^{6}$ S. Iijima, Nature 354, 56 (1991).

${ }^{7}$ J. L. Martins, N. Troullier, and J. H. Weaver, Chem. Phys. Lett. 180, 457 (1991).

${ }^{8}$ B. W. Smith, M. Monthioux, and D. E. Luzzi, Nature 396, 323 (1998).

${ }^{9}$ C. H. Lee, K. T. Kang, K. S. Park, M. S. Kim, H. S. Kim, H. G. Kim, J. E. Fischer, and A. T. Johnson, Jpn. J. Appl. Phys., Part 1 42, 5392 (2003).

${ }^{10}$ S. C. Benjamin et al., J. Phys.: Condens. Matter 18, S867 (2006).

${ }^{11}$ P. Avouris, Z. Chen, and V. Perebeinos, Nat. Nanotechnol. 2, 605 (2007).

${ }^{12}$ Z. Chen, Y.-M. Lin, M. J. Rooks, and P. Avouris, Phys. E 40, 228 (2007).

${ }^{13}$ A. K. Geim and K. S. Novoselov, Nat. Mater. 6, 183 (2007).

${ }^{14}$ B. Xu and X. Chen, Phys. Rev. Lett. 110, 156103 (2013).

${ }^{15}$ M. Xu, S. Ye, and Z. Bačić, J. Chem. Phys. Lett. 6, 3721 (2015).

${ }^{16}$ A. Krachmalnicoff et al., Nat. Chem. 8, 953 (2016).

${ }^{17}$ P. M. Felker and Z. Bačić, J. Chem. Phys. 146, 084303 (2017).

${ }^{18}$ H. A. Gersch and G. C. Knollman, Phys. Rev. 129, 959 (1963).

${ }^{19}$ Q. Wei, S. Kais, and Y. P. Chen, J. Chem. Phys. 132, 121104 (2010).

${ }^{20}$ J. Zhu, S. Kais, Q. Wei, D. Herschbach, and B. Friedrich, J. Chem. Phys. 138, 024104 (2013).

${ }^{21}$ M. Karra, K. Sharma, B. Friedrich, S. Kais, and D. Herschbach, J. Chem. Phys. 144, 094301 (2016).

${ }^{22}$ Q. Wei, Y. Cao, S. Kais, B. Friedrich, and D. Herschbach, ChemPhysChem 17, 3714 (2016).

${ }^{23}$ F. G. S. L. Brandão and G. Gour, Phys. Rev. Lett. 115, 070503 (2015).

${ }^{24}$ T. Halverson and B. Poirier, Chem. Phys. Lett. 624, 37 (2015).

${ }^{25}$ T. Halverson and B. Poirier, J. Phys. Chem. A 119, 12417 (2015).

${ }^{26}$ H.-J. Werner and P. Rosmus, J. Chem. Phys. 73, 2319 (1980).

${ }^{27}$ A. A. Mason and A. H. Nielsen, J. Opt. Soc. Am. 57, 1464 (1967).

${ }^{28}$ R. B. Lehoucq, D. C. Sorensen, and C. Yang, ARPACK Users' Guide: Solution of Large-scale Eigenvalue Problems with Implicitly Restarted Arnoldi Methods (SIAM, 1998).

${ }^{29}$ R. J. Bartlett, Annu. Rev. Phys. Chem. 32, 359 (1981).

${ }^{30}$ P. M. Felker and Z. Bačić, Chem. Phys. Lett. 683, 172 (2017).

${ }^{31}$ D. DeMille, Phys. Rev. Lett. 88, 067901 (2002).

${ }^{32}$ S. F. Yelin, K. Kirby, and R. Côté, Phys. Rev. A 74, 050301 (2006).

${ }^{33}$ M. Ortner, Y. L. Zhou, P. Rabl, and P. Zoller, Quantum Inf. Process. 10, 793 (2011).

${ }^{34}$ M. A. Baranov, M. Dalmonte, G. Pupillo, and P. Zoller, Chem. Rev. 112, 5012 (2012).

${ }^{35}$ B. Abolins, R. Zillich, and K. Whaley, J. Low Temp. Phys. 165, 249 (2011).

${ }^{36}$ B. Abolins, R. Zillich, and K. Whaley, J. Low Temp. Phys. 170, 131 (2013).

${ }^{37}$ C. Herdman, P.-N. Roy, R. Melko, and A. Del Maestro, Phys. Rev. B 89, 140501 (2014).

${ }^{38}$ C. M. Herdman, S. Inglis, P.-N. Roy, R. G. Melko, and A. D. Maestro, Phys. Rev. E 90, 013308 (2014).

${ }^{39}$ C. M. Herdman, P.-N. Roy, R. G. Melko, and A. D. Maestro, Phys. Rev. B 94, 064524 (2016).

${ }^{40}$ C. M. Herdman, P. N. Roy, R. G. Melko, and A. D. Maestro, Nat. Phys. 13, 556 (2017).

${ }^{41}$ Y. N. Kalugina and P.-N. Roy, J. Chem. Phys. 147, 244303 (2017).

${ }^{42}$ M. Srednicki, Phys. Rev. Lett. 71, 666 (1993).

${ }^{43}$ L. Susskind, J. Math. Phys. 36, 6377 (1995).

${ }^{44}$ M. B. Hastings, J. Stat. Mech.: Theory Exp. 2007, P08024.

${ }^{45}$ J. Eisert, M. Cramer, and M. B. Plenio, Rev. Mod. Phys. 82, 277 (2010). 